\title{
A comparative study between anterior lamellar repositioning and anterior lamellar repositioning with blepharoplasty in management of upper eyelid entropion trichiasis
}

\author{
Saad M. Rashad, Mahmoud A. Abd Elhamid, Mahmoud A. El Samkary, and Samah H. El- \\ nenaey \\ Department of Ophthalmology, Faculty of Medicine, Ain Shams University \\ Corresponding author: Mahmoud A. El Samkary, E-Mail: elsamkary76@gmail.com, Phone: 01006282215
}

\begin{abstract}
Background: Entropion is the inward turning of the lid margin that brings both lashes and external keratinized skin into contact with the globe.

Aim of the study: It was to compare the surgical outcome and aesthetic appearance between anterior lamellar repositioning only and anterior lamellar repositioning with blepharoplasty in management of upper eye lid entropion trichiasis.

Patients and Methods: This was prospective interventional study includes 20 lids of 13 patients with upper eyelid cicatricial entropion selected from the ophthalmology outpatient clinic of "Ain Shams University" Hospitals. Patients were randomly divided in 2 groups: group A: Ten lids had an anterior lamellar reposition technique (ALR) with grey line split (GLS) technique. Group B: Ten lids had an anterior lamellar reposition with GLS technique and blepharoplasty. All surgeries were performed by one surgeon (third author) in Ain Shams University hospital.

Results: Our study showed that after 3 month follow up, success rate of first group was $70 \%$, patient satisfaction was $60 \%$ and final aesthetic outcome was 30\% excellent, 30\% fair and $40 \%$ poor results. The second group success rate was $100 \%$, patient satisfaction was $100 \%$ and final aesthetic outcome was $100 \%$ excellent results.

Conclusion: Combining the procedure of ALR with GLS technique with blepharoplasty in surgical correction of upper eye lid cicatricial entropion has excellent both functional and aesthetic outcome and a higher success rate with minimal complication than anterior lamellar reposition only.
\end{abstract}

Keywords: Cicatricial, upper lid, trachoma, anterior lamellar reposition, blepharoplasty, Lid malposition

\section{INTRODUCTION}

Entropion is inward rotation of the eyelid margin. It is one of the most common lid malpositions encountered in clinical practice. There are four types of entropion: congenital, cicatrecial, ivolutional and acute spastic. The pathophysiology of each of these types is different, and so is the treatment ${ }^{(1)}$.

Cicatricial entropion results from a differential horizontal shortening of the posterior lamella of the eyelid in relation to the anterior lamella. Chronic inflammation such as meibomianitis, blepharo-conjunctivitis and trachoma can lead to lid margin keratinization, trichiasis, or dis-tichiasis ${ }^{(1)}$.

Upper cicatricial entropion is usually seen in middle and old aged patients, who may also present with other lid problems, such as dermatochalasis, herniated orbital fat and skin laxity ${ }^{(2)}$.

In Egypt, trachoma is still the most common cause of cicatricial entropion and trichiasis in upper eyelid. The rubbing lashes can cause keratitis, corneal abrasion, ulceration, and even conjunctival metaplasia that can end in blindness and vision loss if left untreated. In addition the irritated spastic orbicularis muscle combined with redundant skin and dermatochalasis further pushes the rubbing lashes downwards making the symptoms more serious and putting the eyelid into a viscous cycle ${ }^{(3)}$.

Anterior lamellar repositioning (ALR) with grey line split technique(GLS) is an effective surgical technique but the excess anterior lamella and dermatochalasis remains a bulk and may slide on the bare tarsus back toward the eyelid margin ${ }^{(4)}$.

Therefore, this procedure can be improved by combining ALR with GLS surgery and blepharplasty, to create a completely penetrating anterior lamellar flap so as to fully shift the anterior lid margin upwards, preventing downward migration of the anterior lamella, improve the surgical outcome and the aesthetic appearance of the procedure with less bulky eyelid appearance ${ }^{(5)}$. Although removal of skin is typically a part of upper lid blepharoblasty but the underlying orbicularis and orbital fat all will play a role in determining the contour of the upper eyelid esthetic unit ${ }^{(6)}$.

\section{AIM OF THE WORK}

The aim is to compare the surgical outcome and aesthetic appearance between anterior lamellar repositioning only and anterior lamellar repositioning 
with blepharoplasty in management of upper eye lid entropion trichiasis.

\section{PATIENTS AND METHODS}

This study is designed as a prospective interventional study. 20 lids of 13 patients (8 female and 5 male) were selected from the Ophthalmology outpatient clinic of "Ain Shams University" Hospitals. Patients were randomly divided in 2 groups: Group A: Ten lids had an ALR with GLS technique. Group B: Ten lids had an ALR with GLS technique and blepharoplasty. Follow up visits was at $1^{\text {st }}$ day, $1^{\text {st }}$ week, $1^{\text {st }}$ month and at 3 months.

Exclusion criteria were: Other types of entropion, lower lid entropion, previous surgery and third degree entropion.

A complete eye and eyelid examination was performed. Extent of entropion was defined according to Kemp and Collin ${ }^{(10)}$ classification: Minimal entropion was defined by posterior migration of the meibomain gland orifices, minimal or incomplete conjunctivalisation of the lid margin, and lashes touching the globe on upgaze. Moderate entropion was diagnosed when, in addition to the early changes detailed above, there was either lash-globe contact in the primary position or thickening of the tarsal plate. Severe lid disturbance can be manifest as a totally disorganized margin with metaplastic lashes and trichiasis, gross lid retraction and keratin formation in plaques on the palpebral conjunctival surface.

Ethical approval: The study was approved by the Ethical Committee of Ain Shams University.

\section{Operative procedure:}

All cases were done under local infiltrative anesthesia \& surface anesthesia. Topical anaesthesia was installed in both eyes. The addressed lid was then infiltrated in the submuscular space by mixture of Mepivacaine HCL 2\% with a 1: 20,000 dilution of Levonoradrenaline (Mepecaine-L).

ALR technique started with grey line split to a depth of 2-3mm across the entire upper eyelid then eye crease incision was made. Dissection of the epitarsal tissues was made from crease incision downwards till root of lashes, if trichiasis affects more than $1 / 3$ of the lid; a complete penetrating flap was done. Several 5/0 vicryl sutures are passed through the skin 1 or $2 \mathrm{~mm}$ above the lashes, through the tarsal plate a few $\mathrm{mm}$ above the skin bite and then back through the skin which when tighten externally evert the lower end of the eyelid. A strip of anterior lamellae about $2 \mathrm{~mm}$ was excised and the skin crease was reformed with interrupted sutures using $6 / 0$ vicryl, taking bites of the underlying levator aponeurosis. The bare area of the posterior lamella was left to granulate.

In the second group, preoperative skin marking was made where the inferior incision was placed 2-3 mm below the intended eyelid-crease across the entire lid and the second incision was made at a variable distance away from the second line, according to the amount of redundant skin to be excised. The redundant skin excised along with two-thirds of the underlying pretarsal fascia to allow definition of the upper lid cleft. The orbital septum was selectively opened in some cases and herniated orbital fat was excised. The rest of ALR steps were done as the first group.

All patients received systemic antibiotics for one week and local antibiotic eye drops for one week. Sutures are left for spontaneous absorption and falling unless infected.

Both groups were followed on day 1 (opening the dressing), week 1 month 1 , and at 3 months. They were asked to return to clinic if they became symptomatic at any time. During the follow-up period the surgical success and complications were evaluated. Success was defined as no lashes touching the globe and the lid margin is parallel to the ocular surface without entropion and ectropion. Assessment of aesthetic outcome is done according to the following; Excellent outcome means success with no residual skin laxity and/or dermatochalasis and no residual post operative complication. Fair outcome means success with residual skin laxity with bulky eyelid appearance and/or dermatochalasis or residual post-operative complication. Poor outcome means recurrent trichiasis and dissatisfied patient. Patient satisfaction was obtained by direct questionnaire to the patient to be satisfied or unsatisfied.

\section{Statistical analysis:}

Data were collected, revised, coded and entered to the Statistical Package for Social Science (IBM SPSS) version 20. Qualitative data were presented as number and percentages while quantitative data were presented as mean, standard 
deviations and ranges when parametric. The confidence interval was set to $95 \%$ and the margin of error accepted was set to $5 \%$. So, the p-value was considered significant as the following: $\mathrm{P}>$ 0.05 was non significant, $\mathrm{P}<0.05$ was significant and $\mathrm{P}<0.01$ was highly significant.

\section{RESULTS}

The patients were randomly divided into two groups; Group1: ALR with GLS technique was performed in 10 eyelids of 7 patients, 3 bilateral and 4 unilateral with mean age was 52.286. Group 2: ALR with GLS technique and blepharoplasty was performed in 10 eyelids of 6 patients, 4 bilateral and 2 unilateral with mean age 50.5 year.

Table (1): Preoperative assessment the two studied groups.

\begin{tabular}{|c|c|c|c|c|}
\hline & & $\begin{array}{c}\text { Group } 1 \\
\text { (10 eyelids) }\end{array}$ & \begin{tabular}{|c|} 
Group 2 \\
$(10$ \\
eyelids)
\end{tabular} & $\begin{array}{c}\text { P-Value } \\
\text { *Significance }\end{array}$ \\
\hline Laterality & \begin{tabular}{|l|} 
Right \\
Left \\
Bilateral
\end{tabular} & $\begin{array}{l}3(42.86 \%) \\
1(14.29 \%) \\
3(42.86 \%)\end{array}$ & $\begin{array}{l}1(16.67 \%) \\
1(16.67 \%) \\
4(66.67 \%)\end{array}$ & 0.585 \\
\hline $\begin{array}{l}\text { Degree of } \\
\text { entropion(10) }\end{array}$ & \begin{tabular}{|l} 
Mild \\
Moderate \\
Sever
\end{tabular} & \begin{tabular}{|c|}
$3(30.00 \%)$ \\
$7(70.00 \%)$ \\
$0(0.00 \%)$
\end{tabular} & \begin{tabular}{|c|}
$3(30.00 \%)$ \\
$7(70.00 \%)$ \\
$0(0.00 \%)$ \\
\end{tabular} & 1.000 \\
\hline $\begin{array}{l}\text { Associated skin } \\
\text { laxity } \\
\text { dermatochalasis }\end{array}$ & $\begin{array}{l}\text { Skin laxity } \\
\text { Dermatochalasis }\end{array}$ & $\begin{array}{l}7(70.00 \%) \\
3(30.00 \%)\end{array}$ & $\left|\begin{array}{l}6(60.00 \%) \\
4(40.00 \%)\end{array}\right|$ & 0.639 \\
\hline
\end{tabular}

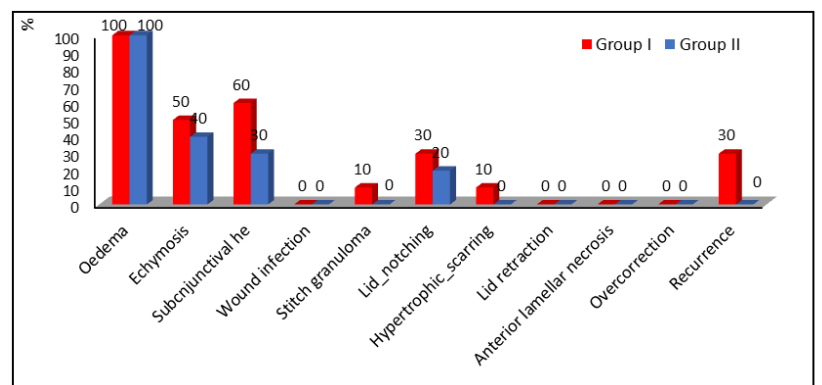

Figure (1): Post-operative complications in the two studied groups.

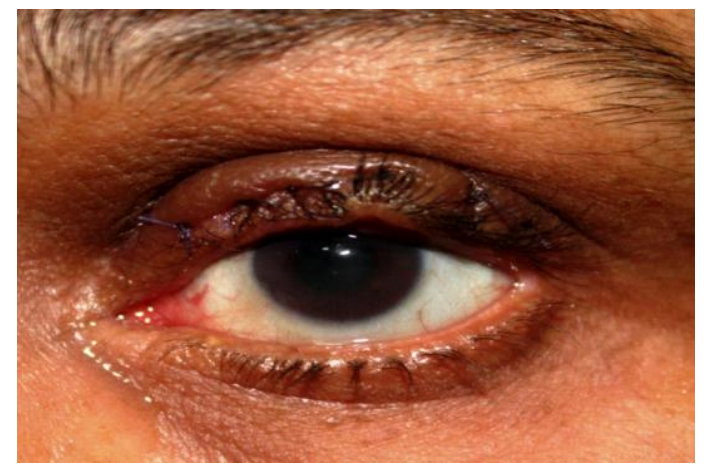

Figure (2): Post-operative lid notching in left eye of female patient in group I.
Table (2): Final results of the studied patients.

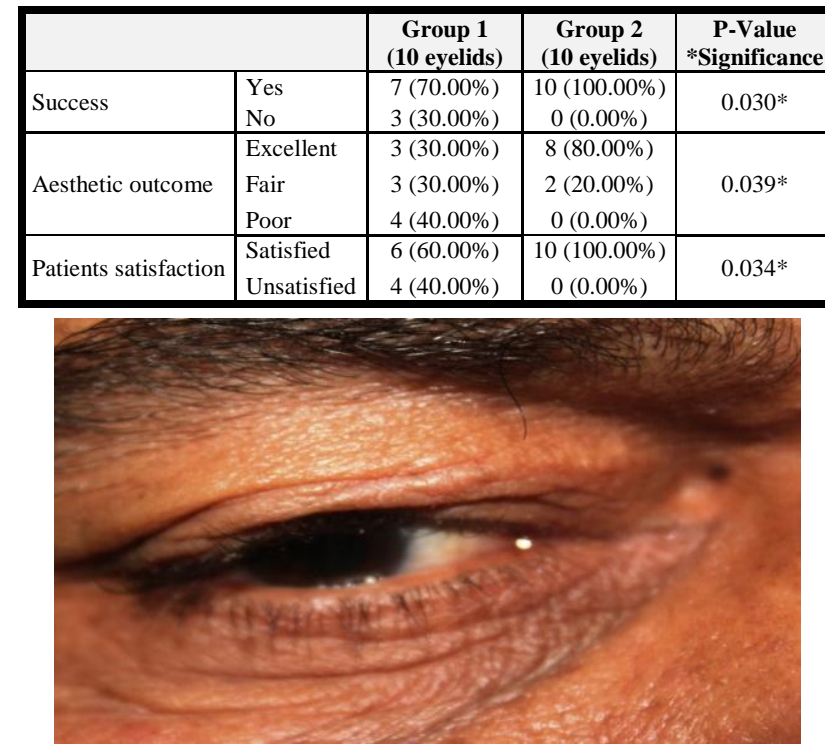

Figure (3): Right eye of male patient in groub 1 showing success after 3 months but note the sagging and the bulky appearance of the eyelid.

\section{DISCUSSION}

Upper cicatricial entropion has a very complex and dynamic mechanism. The anatomic and the physiologic balance between the anterior and the posterior upper eyelid lamellae, keeping the eyelid margin in its normal position, are damaged (7). Posterior lamellar shortening exerts a direct pull on the skin of the lid margin and redirects the anterior lamellar structures, so that the lashes are directed towards the globe. In addition, a number of mechanisms can complicate this entropion process in a damaged eyelid. Elements of laxity or involutional entropion may be combined with those of cicatricial changes ${ }^{(8)}$.

Entropion and trichiasis produce functional disability due to vision loss from corneal opacity. However, even without visual acuity loss; these patients have functional limitations because of photophobia and eye pain, hence the importance of study of different methods of surgical treatment of upper lid cicatricial entropion ${ }^{(9)}$.

Among the various surgical techniques, anterior lamellar reposition with lid margin splitting is one that most respects the anatomy of the upper eyelid and allows precise intraoperative control of eyelid rotation and eversion of the misdirected lashes. Therefore, it reduces significantly the risk of recurrence and significantly enhances the aesthetic results of surgery. It also 
keeps the integrity of the meibomian gland and avoids following iatrogenic dry eye, this seems especially important in trachoma trichiasis cases. The levator aponeurosis and the thick posterior lamella, which maintain the direction of the upper eyelid, are untouched during this procedure ${ }^{(5)}$.

This procedure is performed using various techniques. The procedure has been presented in the literature with different terms including: grey line split with anterior lamellar reposition ${ }^{(10,11)}$, tarso-conjunctival advancement ${ }^{(12)}$; lamellar division $^{(8,13)}$; tarsal advance ${ }^{(18)}$; and anterior lamellar recession ${ }^{(15)}$.

In our ALR technique we depend on splitting the eyelid grey line. This step allows the anterior lamella to retract and gives further space for the lashes to become away from the globe. The surgical procedure of grey line split with vertical anterior lamella repositioning was first described by $\boldsymbol{w e l s} \boldsymbol{h}^{(16)}$, in a case series of 177 patient all with trachomatous trichiasis where after 1 year follow up no patient had returned trichiasis. Numerous authors prefer grey line incision in ALR as Elder and Collin ${ }^{(3)}$, in their series of 16 lids in 11 patients with entropion secondary to ocular cicatricial pemphegoid operated also using this lid split technique. Their series reported five failures (31.2\%). Also Rhatigan et al. ${ }^{(17)}$ reported, in a study of 28 lids of 19 patient with follow up time 10-34 months (mean 16 months) where all patient had the same surgical procedure of ALR with grey line split technique,success rate of $24 \operatorname{lids}(85 \%)$.

In our ALR technique, a lid crease incision was made. Interlamellar separation can be performed through lid margin or eyelid crease approach as done by Elder and Collin ${ }^{(3)}$, Rhatigan et al. ${ }^{(17)}$, Dhaliwal et al. ${ }^{(18)}$. Ross et al. ${ }^{(19)}$ and as performed in this study or dissection can be done by both incisions as done by Barr et al. ${ }^{(20)}$. We believe that making lid crease incision and dissection through it down to the lid margin is more effective for the following reasons; first, the sub-orbicularis dissection can be easily performed until riolan's muscle is reached and absorbable everting sutures can be passed internally. Second, unidentifiable grey line may present in some cases. Third, it allows for addressing concurrent dermatochalasis and other lid malpositions. Finally, lid crease formation with bites through the levator helps maintain an upward vector of traction to the anterior lamella and lash eversion.
Not all the authors prefer lid crease incision, starting from welsh ${ }^{(16)}$ itself, where his description did not involve lid crease incision, however, he made a vertical cut along the anterior lamella at lateral canthal area $4 \mathrm{~mm}$ away the lash line where excess skin is trimmed and the anterior lamellae is pulled and sutured with posterior lamellae using non absorbable silk suture. Sodhi et $\boldsymbol{a l}{ }^{(11)}$ separated the two lamellae from the grey line up to the upper border of the tarsus with no skin crease incision. They additionally made $2-3 \mathrm{~mm}$ vertical incision through the medial $(1 \mathrm{~mm}$ lateral to the lacrimal punctum) and lateral to anterior lid margin of the tarsal plate to aid lid splitting in to anterior and posterior lamella. They reported success rate was $88.4 \%$ in their study of 84 eyelids and follow up time 12 months. Ahmed and Abdelbaky ${ }^{(21)}$ in a large study with 752 eye lids of 445 patients with mean age 53, ALR with grey line split was performed with also no crease incision. They reported by the end of 6 months follow up, $14.9 \%$ of lids showed recurrence while $66.1 \%$ were completely cured and $19 \%$ had partial success.

We leave the bare posterior lamella to granulate. Although leaving the tarsus bare for spontaneous epithelialization gave the patient an odd appearance in the beginning post-operative period, we feel that this step is avoidable as the raw tarsal surface heals well even when it is left uncovered. Some surgeons advocate covering the raw tarsal surface with a graft. A graft placed on the anterior surface of the tarsus is believed to act as a spacer or barrier to help in preventing repeat displacement of lashes toward the eyelid margin.

Steinkoger ${ }^{(22)}$ repaired severe upper eyelid entropion with trichiasis with ALR with GLS technique and used either autologous skin graft or mucous-membrane graft. Kemp and Collin ${ }^{(8)}$ in some cases after lamella division and posterior tarsoconjunctival advancement, the bare area of tarsus was covered with an autogenous graft of mucous membrane. Chen et al. (23) adopted a modified grey line splitting with tarsal wedge resection and acellular human dermal allograft in treating cicatricial entropion due to chemical and thermal injuries.

We made five vicryl $5 / 0$ everting sutures placed through the skin and orbicularis muscle and tied to evert the lashes. Some surgeon passed recessing sutures directly through the anterior lamella to the anterior surface of the tarsus as 
Kemp and Collin ${ }^{(8)}$ and Owji and Tehrani ${ }^{(24)}$. Other surgeon had to pass tarsal traction sutures to prevent retraction of the posterior lamella in their patient but different technique descried. Sodhi et al. ${ }^{(11)}$ in their study made their 3 recessing transverse mattress sutures using 6/0 chromic cat gut, traversed the full thickness of the lid at the level of the fornix posteriorly, pulling the posterior lamella forward and then back to the skin again 2 $\mathrm{mm}$ from the lash line. Kuckelkorn et al. ${ }^{(12)}$ reported ALR with fornix suture and fixing the tarsal traction suture to the cheek.

In prescence of lid laxity and dermatochalasis even a small amount of conjunctival scarring can cause entropion. Mechanically this may affect the position of the lashes resulting in worsening of the entropion ${ }^{(17,11)}$ with the ALR procedure alone, the redundancy of the upper eyelid skin and the underlying orbicularis muscle may be aggravated. Hence, excess skin and muscle removal through blepharoplasty has been recommended to prevent downward migration of the anterior lamella and provide a less bulky postoperative eyelid ${ }^{(5,10,25)}$. Tie et al. ${ }^{(10)}$ combined ALR with amniotic membrane transplantation and blepharoblasty, a total of 25 lid procedures was performed, the mean follow-up period was 17.8 months, the procedure was a complete success for 22 lids (88\%). Bi et al. ${ }^{(5)}$ combined anterior lamellar reposition with blepharoplasty and lid split surgery creating a completely penetrating flap. Of 144 eyelids operated and followed up for at least 12 months, the recurrence rate was $0 \%$ at 3 monthes, $2.8 \%$ at 6 months and $5.6 \%$ at 12 months. There was no case of secondary lid retraction or ectropion and the aesthetic alteration were accepted by all patient. Also Aghai et al. ${ }^{(25)}$ combined ALR with blepharoblasty and supratarsal fixation. Follow up time was at least 12 months with mean 21.06. Success was observed in $39(75 \%)$ and failure in $13(25 \%)$ in which eight eyelids $(15.4 \%)$ had less than five trichiatic eyelashes and the rest had recurrence of entropion.

In our opinion, the long-standing eyelid entropion and trichiasis is often combined with vertical eyelid skin redundancy and the pretarsal portion of orbicularis muscle spasmus and hypertrophy. In addition protrouding orbital fats will aggrevate the condition. Obviously, the ALR procedure alone will further aggravate the redundancy of the upper eyelid skin and its subtus orbicularis muscle.

ALR with blepharoplasty is an excellent choice to manage proplem entropion cases

with mixed cicatricial and laxity components. It addresses several factors relating to upper eyelid entropion triachiasis such as: complete reposition of lid margin lashes, orbital fat prolapse correction, dermatochalasis correction and release of thick spasmus orbicularis muscle. Most importantly, after these steps, the anterior lamella shifted upwards rests on its new bed, with little tendency to shift downwards, and the re-epithelized epidermis would smoothly cover the exposed tarsus several days later.

\section{CONCLUSION}

Combining the procedure of ALR with GLS technique with blepharoplasty in surgical correction of upper eye lid cicatricial entropion has excellent both functional and aesthetic outcome and a higher success rate with minimal complication

Limitations: A potential design limitation in our study is the short postoperative follow-up time and the small sample size.

\section{RECOMMENDATION}

We recommend further studies with longer follow up intervals and larger sample size.

\section{REFERENCES}

1. Gladstone GJ, Black EH (2002): entropion, In: Oculoplastic surgery atlas; Springer, 2: 1727.

2. Collin JR (1991): Blepharochalasis, A review of 30 cases. Ophthal. Plast. reconstr. Surgery, 7:153-7.

3. Elder MJ and Collin R (1996): Anterior lamellar repositioning and grey line split for upper eyelid entropion in ocular cicatricial pemphigoid. Eye, 10:439-42.

4. Hintschich CR (1997): Anterior lamellar repositioning "for correction of entropion of the upper eyelid. Ophthalmologe, 94:436-40.

5. Bi YL, Zhou Q (2009): Anterior lamellar repositioning with complete lid split: a modified method for treating upper eyelids trichiasis in Asian patients. JPARS., 62:1395402. 
6. Goldberg RA, Hwang CJ, Li TG and Schorr N (2012): Blepharoplasty In: Speath GL, Danesh-meyer HV, Golgberg I and Kampik A; Ophthalmic surgery, 51:385-398.

7. Guzey M, Ozardali I, Basar E et al. (2000): Survey of trachoma: the histopathology and the mechanism of progressive cicatrization of eyelid tissues. Ophthalmologica, 214(4):277284.

8. Kemp EG, Collin JR (1986): Surgical management of upper lid entropion. British Journal of Ophthalmology, 70; 575-579.

9. Dhaliwal U, Monga PK, Gupta VP (2004): Comparison of three surgical procedures of differing complexity in the correction of trachomatous upper lid entropion: A prospective study. Orbit., 23: 227-236.

10. Ti SE, Tow SL, Chee SP (2001): Amniotic membrane transplantation in entropion surgery. Ophthalmology, 108: 1209-1217.

11. Sodhi PK, Yadava U, Pandey RM, Mehta DK (2002): Modified grey line split with anterior lamellar repositioning for treatment of cicatricial lid entropion. Ophthalmic Surgery and Lasers,, 33: 2: 169-174.

12. Kuckelkorn R, Schrage N, Becker J, Reim $M$ (1997): Tarsoconjunctival advancement: a modified surgical technique to correct cicatricial entropion and metaplasia of the marginal tarsus. Ophthalmic Surg Lasers, 28(2):156-161.

13. Sodhi PK, Yadava U, Mehta DK (2002): Efficacy of lamellar division for correcting cicatricial lid entropion and its associated features unrectified by the tarsal fracture technique. Orbit, 21: 9-17,

14. Reacher MH, Huber MJ, Canagaratnam $R$ et al. (1990): A trial of surgery for trichiasis of the upper lid from trachoma. Br J Ophthalmol., 74:109-13.

15. Koreen IV, Taich A, Elner VM (2009): Anterior lamellar recession with buccal mucous membrane grafting for cicatricial entropion. Ophthal Plast Reconstr Surg., 25: $180-4$.

16. Welsh NH (1969): The treatment of cicatricial entropion. S Afr Med J., 43: 172-5.
17. Rhatigan M, Ashworth J,Goodall $K$ and Leatherbarow B (1997): Correction of blepharoconjunctivitis-Related upper eyelid entropion using the anterior lamellar reposition technique. Eye, 11:118-120.

18. Dhaliwal U, Monga PK, Gupta VP (2004): Comparison of three surgical procedures of differing complexity in the correction of trachomatous upper lid entropion: a prospective study. Orbit, 23: 227-36.

19. Ross AH, Cannon PS, Selva D, Malhotra $R$ (2011): Management of upper eyelid cicatricial entropion. Clinical and Experimental Ophthalmology,39: 526-536.

20. Barr K, Essex RW, Liu S, and Henderson T (2014): Comparison of trichiasis recurrence after primary bilamellar tarsal rotation or anterior lamellar repositioning surgery performed for trachoma. Clinical \& Experimental Ophthalmology, 42: 4: 311-316.

21. Ahmed RA, Abdelbaky SH (2015): Short Term Outcome of Anterior Lamellar Reposition in Treating Trachomatous Trichiasis, Clinical Study, Journal of Ophthalmology, Hindawi Publishing Corporation.

22. Steinkogler FJ (1986): Treatment of upper eyelid entropion. Eyelid split surgery and fibrin sealing of free skin transplants. Ophthal Plast Reconstr Surg., 2: 183-187.

23. Chen J, Wang Z, Gu J (2008): Management of cicatricial entropion of the upper eyelid using acellular human dermal allograft. J Plast Reconstr Aesthet Surg., 61: 610-614.

24. Owji N, Tehrani MJ (2013): Anterior lamellar recession in the management of the trachomatous cicatricial entropion of the upper eyelids: Outcomes and indications. Asian Journal of Ophthalmology, 13(2):42-47.

25. Aghai GH, Gordiz A, et al. (2016): Anterior lamellar recession, blepharoplasty, and supratarsal fixation for cicatricial upper eyelid entropion without lago-phthalmos. Eye, 30 (4): 627-631. 